\title{
The effect of nonlinear ionospheric conductivity enhancement on magnetospheric substorms
}

\author{
E. Spencer and S. Patra \\ Electrical and Computer Engineering Department, Utah State University, Logan, UT 84322, USA \\ Correspondence to: E. Spencer (edmund.spencer@usu.edu) \\ Received: 10 October 2012 - Revised: 30 January 2013 - Accepted: 16 May 2013 - Published: 27 June 2013
}

\begin{abstract}
We introduce the effect of enhanced ionospheric conductivity into a low-order, physics-based nonlinear model of the nightside magnetosphere called WINDMI. The model uses solar wind and interplanetary magnetic field (IMF) parameters from the ACE satellite located at the L1 point to predict substorm growth, onset, expansion and recovery measured by the AL index roughly $50-60 \mathrm{~min}$ in advance. The dynamics introduced by the conductivity enhancement into the model behavior is described, and illustrated through using synthetically constructed solar wind parameters as input. We use the new model to analyze two well-documented isolated substorms: one that occurred on 31 July 1997 from Aksnes et al. (2002), and another on 13 April 2000 from Huang et al. (2004). These two substorms have a common feature in that the solar wind driver sharply decreases in the early part of the recovery phase, and that neither of them are triggered by northward turning of the IMF Bz. By controlling the model parameters such that the onset time of the substorm is closely adhered to, the westward auroral electrojet peaks during substorm expansion are qualitatively reproduced. Furthermore, the electrojet recovers more slowly with enhanced conductivity playing a role, which explains the data more accurately.
\end{abstract}

\section{Introduction}

The substorm is a fundamental geomagnetic process in the earth's magnetosphere that has been a topic of intense research over several decades. Of particular interest is how the ionosphere, inner magnetosphere, and geotail dynamics influence the growth, onset, expansion and recovery phases of a typical substorm. These components may interact differently under varying solar wind conditions, or different classes of substorm activity. Currently, there are three accepted classes of substorms: isolated substorms, storm time substorms, and sawtooth events also known as periodic substorms (Partamies et al., 2009).

In this work, we modify the equations of a low-order, physics-based nonlinear model of the magnetosphere called WINDMI (Horton and Doxas, 1996; Spencer et al., 2007), in order to account for the contribution of ionospheric conductivity enhancement to substorm behavior. The current version of the model is available at the NASA Community Coordinated Modeling Center for near real time forecasts of space weather activity (Mays et al., 2009).

The standard explanation for ground-based observations of substorm development is through a nonlinear energy loading and unloading process (Baker et al., 1999). The growth phase occurs when the interplanetary magnetic field (IMF) turns southward for a period of time, and plasma sheet thinning develops. Then reconnection occurs, followed by dipolarization. This growth phase is not always observable because of the fluctuations in the IMF. The precise mechanisms for substorm onset are still under investigation (Sergeev et al., 2012), but reconnection in the tail is understood to be the point when the dipolarization occurs, which marks the expansion phase of a substorm. Finally, as the plasma energy is lost, the substorm goes into its recovery phase. We note that a strongly fluctuating solar wind may trigger bursts in the auroral electrojet (AE) index (Pulkkinen et al., 2006), but this effect is not represented in the nonlinear dynamical WINDMI model.

The modeling of nonlinear loading and unloading behavior of geomagnetic substorms under southward IMF conditions has been explored by several authors (Klimas et al., 1992, 1996; Blanchard and McPherron, 1993). Vassiliadis et al. (1995) used a nonlinear filter approach for describing 
the solar wind magnetosphere coupling, and further to predict the AL index (Klimas et al., 1998). Weigel et al. (1999) used a neural network approach to predicting AL index activity. The WINDMI model was used by Horton et al. (2003) to classify substorms into 3 categories, one of which is the northward turning triggered substorm (Gallardo-Lacourt et al., 2012). Here we continue to develop the WINDMI model as a tool for analyzing and forecasting the loadingunloading type of substorm.

A detailed photochemical equilibrium model of the quiet time ionosphere that depended on the solar zenith angle and F10.7 flux was developed by Rasmussen et al. (1988). An ionospheric conductance model based on ground magnetic disturbance data was developed by Ahn et al. (1998). The dependence of ionospheric conductivity on energy deposited by precipitating electrons was modeled by Robinson et al. (1987). It is known that the ionospheric conductivity is enhanced during the growth and expansion periods of a substorm, when increased particle precipitation occurs in the high latitude ionosphere parallel to the magnetic field lines, reported by Tang et al. (2011) and references therein. Aksnes et al. (2002) derive conductance maps showing the increase in Hall and Pedersen conductivities during an isolated substorm using data from the Polar Ionospheric X-ray Imager (PIXIE) and the Ultraviolet Imager (UVI) on board the Polar satellite. Gjerloev and Hoffman (2000a) calculated the height-integrated Hall and Pedersen conductances during substorms using the Dynamics Explorer 2 data. In our model we introduce the effect of conductivity enhancement by including a nonlinear conductivity term controlled by the parallel streaming kinetic energy of the plasma along magnetic field lines that terminate on the ionosphere. This term adds a new component to the dynamics of substorms, which we explore.

Our aim in this paper is to determine how the enhanced ionospheric conductivity plays a role in the buildup and recovery of a substorm. For instance, if the conductivity rises slowly during the growth phase, and then is suddenly enhanced in a sharp manner at substorm onset, the resulting increase in the auroral electrojet current appears more sawtooth-like, as opposed to the case of the electrojet signature due to a reconnection trigger only. The faster the rate of enhanced conductivity, the more sawtooth-like the response becomes. We also evaluate the model results for two isolated substorm events: one on 31 July 1997, and another on 13 April 2000. These two substorms are examples of welldocumented events, where in Aksnes et al. (2002), the onset, expansion and recovery phases are clear while ionospheric conductance enhancement is clearly present, and, in Huang et al. (2004), the onset of the expansion phase of the substorm is timed accurately. These two substorms also have two common features in that the solar wind driver sharply decreases in the early part of the recovery phase, and that neither of them are triggered by northward turning of the IMF Bz. The model behavior with enhanced conductivity is compared to the case where the ionosphere conductivity is constant.

In the next section we describe the WINDMI model. In Sect. 3, we introduce a nonlinear conductance term into the model. In Sect. 4 we show how the increased conductivity affects the phases of a synthetic isolated substorm. In Sect. 5 we compare the model to data for the two substorm events. We then summarize the paper and conclude with some suggestions for future work.

\section{WINDMI Model}

The plasma-physics-based WINDMI model uses the solar wind dynamo voltage $V_{\mathrm{sw}}$ generated by a particular solar wind-magnetosphere coupling function to drive eight ordinary differential equations describing the transfer of power between the major energy components of the nightside magnetosphere. The WINDMI model is described in some detail in Doxas et al. (2004), Spencer et al. (2007) and more recently in Patra et al. (2011). The equations of the model are given by the following:

$$
\begin{aligned}
L \frac{d I}{\mathrm{~d} t} & =V_{\mathrm{sw}}(t)-V+M \frac{d I_{1}}{\mathrm{~d} t} \\
C \frac{d V}{\mathrm{~d} t} & =I-I_{1}-I_{\mathrm{ps}}-\Sigma V \\
\frac{3}{2} \frac{d p}{\mathrm{~d} t} & =\frac{\Sigma V^{2}}{\Omega_{\mathrm{cps}}}-u_{0} p K_{\|}^{1 / 2} \Theta\left(u_{1}\right)-\frac{p V A_{\mathrm{eff}}}{\Omega_{\mathrm{cps}} B_{\mathrm{tr}} L_{y}}-\frac{3 p}{2 \tau_{E}} \\
\frac{d K_{\|}}{\mathrm{d} t} & =I_{p s} V-\frac{K_{\|}}{\tau_{\|}} \\
L_{I} \frac{d I_{1}}{\mathrm{~d} t} & =V-V_{I}+M \frac{d I}{\mathrm{~d} t} \\
C_{I} \frac{d V_{I}}{\mathrm{~d} t} & =I_{1}-I_{2}-\Sigma_{I} V_{I} \\
L_{2} \frac{d I_{2}}{\mathrm{~d} t} & =V_{I}-\left(R_{\mathrm{prc}}+R_{A 2}\right) I_{2} \\
\frac{d W_{\mathrm{rc}}}{\mathrm{d} t} & =R_{\mathrm{prc}} I_{2}^{2}+\frac{p V A_{\mathrm{eff}}}{B_{\mathrm{tr}} L_{y}}-\frac{W_{\mathrm{rc}}}{\tau_{\mathrm{rc}}}
\end{aligned}
$$

The nonlinear equations of the model trace the flow of electromagnetic and plasma mechanical energy through eight pairs of transfer terms. The remaining terms describe the loss of energy from the magnetosphere-ionosphere system through plasma injection, ionospheric losses and ring current energy losses.

In the differential equations, the coefficients are physical parameters of the magnetosphere-ionosphere system. The quantities $L, C, \Sigma, L_{I}, C_{I}$ and $\Sigma_{I}$ are the magnetospheric and ionospheric inductances, capacitances, and conductances. $A_{\text {eff }}$ is an effective aperture for particle injection into the ring current, which on the dusk side merges with what is known as the Alfvén layer (Doxas et al., 2004). The resistances in the partial ring current and region 2 current $I_{2}$ are $R_{\mathrm{prc}}$ and $R_{A 2}$ respectively, and $L_{2}$ is the inductance 
of the region 2 current. The coefficient $u_{0}$ in Eq. (3) is a heat flux limiting parameter. The energy confinement times for the central plasma sheet, parallel kinetic energy and ring current energy are $\tau_{E}, \tau_{k}$ and $\tau_{r c}$ respectively. The effective width of the magnetosphere is $L_{y}$, and the transition region magnetic field is given by $B_{\mathrm{tr}}$. The pressure-gradient-driven current is given by $I_{\mathrm{ps}}=L_{x}\left(p / \mu_{0}\right)^{1 / 2}$, where $L_{x}$ is the effective length of the magnetotail. The outputs of the model are the AL and Dst indices, in addition to the magnetospheric field-aligned currents.

The pressure unloading function $\Theta\left(u_{1}\right)=\frac{1}{2}\left[1+\tanh u_{1}\right]$ where $u_{1}=\left(I-I_{c}\right) / \Delta I$ in Eq. (3) is specified by a critical current $I_{c}$ and the interval $\Delta I$ for the transition to loss of plasma along newly opened magnetic field lines with a parallel thermal flux $q_{\|}$. It changes from zero to unity as a function of $I$ compared to $I_{c}$. The unloading function follows from current-gradient-driven tearing modes or cross-field current instabilities, as described in Yoon et al. (2002).

The AL index is obtained from the region 1 current $I_{1}$ by assuming a constant of proportionality $\lambda_{\mathrm{AL}}[A / n T]$, giving $\Delta B_{\mathrm{AL}}=-I_{1} / \lambda_{\mathrm{AL}}$. The input function used for the model is the standard rectified $v B_{s}$ formula (Reiff and Luhmann, 1986), given by

$V_{\mathrm{sw}}^{B s}=40(k V)+v_{\mathrm{sw}} B_{s}^{\mathrm{IMF}} L_{y}^{\mathrm{eff}}(k V)$,

where $v_{\mathrm{sw}}$ is the $\mathrm{x}$-directed component of the solar wind velocity in GSM coordinates, $B_{s}^{\mathrm{IMF}}$ the southward IMF component and $L_{y}^{\text {eff }}$ an effective cross-tail width over which the dynamo voltage is produced. For northward or zero $B_{s}^{\mathrm{IMF}}$, a nominal viscous voltage of $4 \mathrm{kV}$ is used to drive the system.

\section{Enhanced nonlinear conductivity during substorm growth and onset}

The ionosphere electron density in the auroral zone is strongly affected by particle precipitation along magnetic field lines. Impact ionization increases with the number and energy of particles entering the ionosphere down to 100$120 \mathrm{~km}$, where the auroral electrojet currents flow. The magnetic field lines begin in the magnetotail plasma sheet and close in the ionosphere above 65 degrees latitude. The conductivity is composed of the Hall and Pedersen components, given by Coumans et al. (2004):

$\sigma_{P}=\frac{N_{e} e}{B}\left(\frac{v_{\mathrm{en}} \omega_{\mathrm{ce}}}{v_{\mathrm{en}}^{2}+\omega_{\mathrm{ce}}^{2}}+\frac{\nu_{\mathrm{in}} \omega_{\mathrm{ci}}}{v_{\mathrm{in}}^{2}+\omega_{\mathrm{ci}}^{2}}\right)$
$\sigma_{H}=\frac{N_{e} e}{B}\left(\frac{\omega_{\mathrm{ce}}^{2}}{v_{\mathrm{en}}^{2}+\omega_{\mathrm{ce}}^{2}}-\frac{\omega_{\mathrm{ci}}^{2}}{v_{\mathrm{in}}^{2}+\omega_{\mathrm{ci}}^{2}}\right)$

where $N_{e}$ is the electron density, $e$ the electron charge, $v_{\mathrm{en}}$ and $v_{\text {in }}$ are the electron neutral and ion neutral collision frequencies, and $\omega_{\mathrm{ce}}$ and $\omega_{\mathrm{ci}}$ are the electron and ion gyrofrequencies respectively in the geomagnetic field $B$.
The Pedersen component is the conductivity parallel to the electric field and perpendicular to the magnetic field. The Hall component is the conductivity perpendicular to both the electric and magnetic fields. The conductivity is a strong function of $N_{e}$. Ionization is expected to ramp up due to increased precipitation, and saturates as the ionization rate is balanced by recombination and losses. In addition, the conductivity increases or decreases according to the electron neutral collision frequency.

When the plasma sheet electric field is enhanced during the substorm growth phase, the cross-tail electric field drives field-aligned currents from the plasma sheet into the ionosphere. As the upward field-aligned currents in the midnight to pre-midnight sector intensify, parallel electric potentials form above the auroral ionosphere that accelerate charged particles into the ionosphere (Birn et al., 2012; Knight, 1973). There is a sudden increase in the parallel electric fields around substorm onset (Morioka et al., 2010), which should increase the rate of particle precipitation. Gjerloev and Hoffman (2000b) and Aksnes et al. (2002) report an increase in the Hall and Pedersen conductivities in the ionosphere at auroral latitudes during substorm activity. The strength of the westward auroral electrojet will consequently be increased since it is controlled by the Hall conductivity (Akasofu, 2004).

To include the effect of a nonlinear ionospheric conductance that is enhanced through increased particle precipitation during the substorm growth and expansion phases, Eq. (6) is modified with a nonlinear function controlled by the parallel kinetic energy along field lines $K_{\|}$. The equation then takes the form

$C_{I} \frac{d V_{I}}{d t}=I_{1}-I_{2}-\left(\Sigma_{I}+\Sigma_{\mathrm{enh}} \Theta\left(u_{2}\right)\right) V_{I}$.

The function $\Theta\left(u_{2}\right)$ is in the same form as the function in Eq. (3) except that in this case $u_{2}=\left(K_{\|}-K_{\|}^{0}\right) / \Delta K$, where $K_{\|}^{0}$ is a lower limit for the parallel kinetic energy above which the conductivity becomes enhanced at the altitude of the auroral electrojet. We expect that below this value of $K_{\|}$ the precipitating particles do not penetrate to the lowest altitudes. The parameter $\Delta K$ controls the rate of conductivity enhancement up to a saturation level of $\Sigma_{I}+\Sigma_{\text {enh. }}$. Physically this parameter is governed by the ionization efficiency at a particular altitude.

\section{Implications on substorm dynamics}

If the ionospheric conductivity is assumed to be constant during the growth, expansion and recovery phases of a substorm, the westward auroral electrojet current signature is driven only by solar wind magnetosphere coupling, and magnetospheric dynamics. This is illustrated with the black curve in Fig. 1 where we run the model with a southward IMF of $20 \mathrm{nT}$, and solar wind velocity of $600 \mathrm{~km} \mathrm{~s}^{-1}$, for $20 \mathrm{~min}$. 
We see the increase in the current during the growth phase of the substorm when the geotail magnetic field stretches, the substorm onset, followed by the expansion phase, and finally the recovery phase. The sudden surge in auroral electrojet current is caused by the unloading trigger $\Theta\left(u_{1}\right)$ being switched on corresponding to reconnection in the geomagnetic tail.

In the same figure, we also show how the input into the model creates an increase in the auroral electrojet current depending on the rate of ionosphere conductivity enhancement, shown as a red curve. In this case, the reconnection switch is turned off; the surge is entirely controlled by the enhanced conductivity. We have slightly exaggerated the enhanced conductivity following Tang et al. (2011). The enhancement is sharply increased when the precipitation rate crosses a threshold level, which likely occurs sometime during the later part of the growth phase of a substorm. This corresponds to the idea that, above a certain level of activity, the precipitating particles penetrate deeply enough into the ionosphere to reach the altitudes where the auroral electrojet flows. This type of enhancement causes a surge and recovery of the electrojet similar to the charge-discharge process of a linear RL circuit.

Lastly, we now include both the reconnection trigger as well as the conductivity enhancement. This is shown with the blue curve in Fig. 1. Now the overall substorm event is characterized by both the effects simultaneously affecting the expansion and recovery phases. Of particular note is that, in the later part of the recovery, the electrojet settles smoothly to rest without the negative overshoot. We also note that the overall level of electrojet activity increases when the conductivity is enhanced.

To examine the behavior of the model for changes in the value of $K_{\|}^{0}$ and $\Sigma_{\text {enh }}$, we chose upper and lower values of each parameter around the nominal values whose results are shown in Fig. 1. We do this for the case when both the reconnection switch and conductivity enhancement are present. In the nominal case, which corresponds to the blue curve in Fig. 1, we have $K_{\|}^{0}=6 \times 10^{13} \mathrm{~J}, \Sigma_{\text {enh }}=10 \mathrm{~S}$, and $\Delta_{K}=1.5 \times 10^{13} \mathrm{~J}$. With changes in parameter values above and below these nominal values, the character of the output auroral electrojet current is altered as shown in Fig. 2. The input is kept the same as that in Fig. 1.

The behavior is as expected. In the top panel of Fig. 2, we see that, as the threshold level for beginning the enhancement is lowered, the field-aligned currents increase faster during the growth period of a substorm, and decay more slowly during the recovery phase. In the second panel of Fig. 2, it is clear that if the upper saturation level of the ionospheric conductivity is increased, we obtain much stronger current values. Finally, in the bottom panel of Fig. 2, the effect of $\Delta_{K}$ can be observed to change the rate of increase of the conductivity around the threshold level $K_{\|}^{0}$, resulting in a change of the rate of increase and decrease of the electrojet strength.
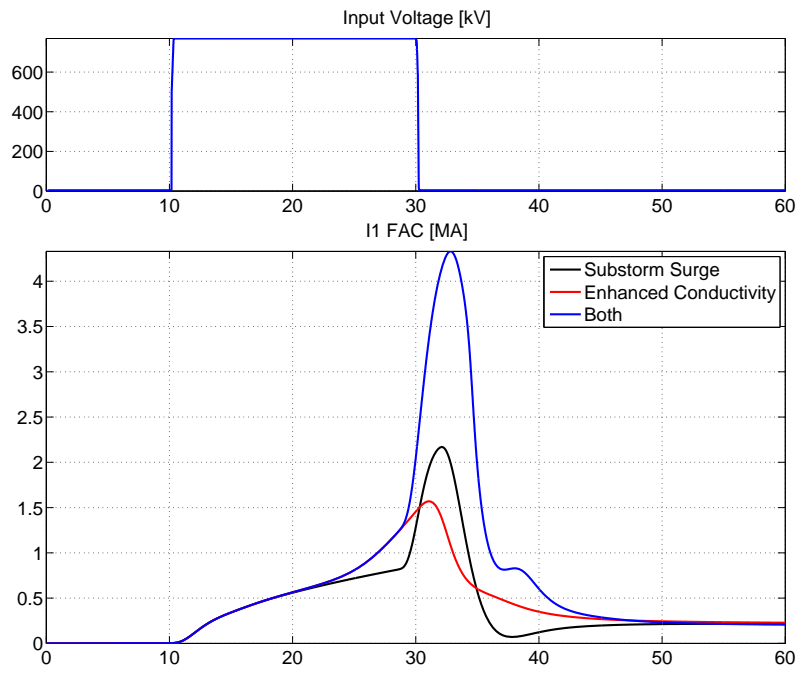

Fig. 1. The response of the magnetosphere-ionosphere field-aligned current system simulated by the WINDMI model for the case with a reconnection switch (shown in black), or the enhanced conductivity (shown in red), or both (shown in blue).

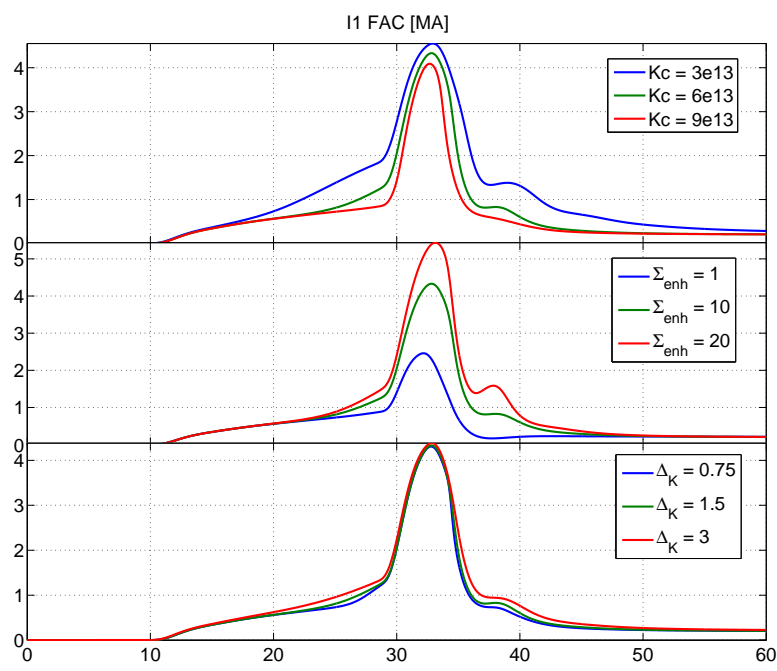

Fig. 2. The response of the WINDMI model when the values of $K_{\|}^{0}, \Sigma_{\text {enh }}$ and $\Delta_{K}$ are varied refers to the nominal plot shown by the blue curve in Fig. 1. The threshold parallel kinetic energy above which the conductivity becomes strongly enhanced is varied $3-9 \times$ $10^{13} \mathrm{~J}$, the saturation level of the conductivity is varied in the range of $1-20 \mathrm{~S}$, and the rate of increase in the conductivity is varied in the range of $0.75-3 \times 10^{13} \mathrm{~J}$.

\section{Results for 31 July 1997 and 13 April 2000 substorms}

We first use the new model to analyze an isolated substorm that occurred between 03:00 and 04:00 UTC on 13 April 2000. The growth phase of this substorm is not clear from the data. The onset of the substorm was between 
03:05 and 03:10 UTC on the day (Huang et al., 2004). With the solar wind parameters obtained from the ACE spacecraft translated into the nose of the magnetosphere (Spencer et al., 2007), the resultant input rectified voltage is shown in the bottom panel of Fig. 3.

In order to obtain our results, we adjust our model in order to fulfill two criteria. The first is that the onset time must be almost at the onset time reported by Huang et al. (2004). The second is that we try to capture the overall substorm growth, expansion and recovery phases. A particularly important reason why this substorm was chosen is because the input voltage decreases sharply at approximately 03:30 UTC, which means that the observed recovery phase is not affected by solar wind driving. In addition, the IMF Bz remains southward during the onset, ensuring that this substorm is not a case that is possibly driven by northward turning of the IMF.

In the first case, the model parameters are adjusted to perform as well as possible on the substorm, but without the conductivity enhancement. The result is shown by the red curve in Fig. 3. In the plot, the AL index refers to the lower auroral electrojet index obtained from the World Data Center for Geomagnetism, Kyoto, Japan. These data represent the deflection of the earth's magnetic field at auroral latitudes from 12 stations (http://wdc.kugi.kyoto-u.ac.jp/). The time scale is in minutes starting from 00:00 UTC. The model run shows the growth phase, onset at roughly the same time as observed, and a fast expansion followed by recovery after 03:20 UTC. The recovery rate is fast in the model, faster than the activity shown in the data.

In the second case, we show how the model result changes when the enhancement in the ionospheric conductivity is included. This is shown by the green curve in Fig. 3. For this run the model parameters are adjusted differently in order to capture the substorm activity. In this case the expansion phase begins at the same time as when the enhanced conductivity is not used, but the peak activity occurs a little later (03:28 UTC) and the recovery phase is much slower. Here the recovery appears to follow the recovery phase in the data more closely, except during the time period 03:20 UTC to 03:40 UTC, where a decline in AL is followed by a small surge in the data. We note that this second surge in AL that occurs around 03:38-03:40 UTC does not appear to be driven by the solar wind. This is because the solar wind activity has subsided by this time.

Overall, the model captures the substorm activity, but interpretation of the result depends on whether enhanced conductivity is assumed to play a role or not. A quantitative measure that we use to determine the goodness of fit between the model output and the data is the average relative variance (ARV). For the 13 April 2000 substorm, the ARV when only a reconnection trigger is used is 0.71 . When the conductivity enhancement is also included, the ARV is 0.32. Using this measure, the lower the ARV the better the performance, which suggests that the enhanced conductivity improves the result.

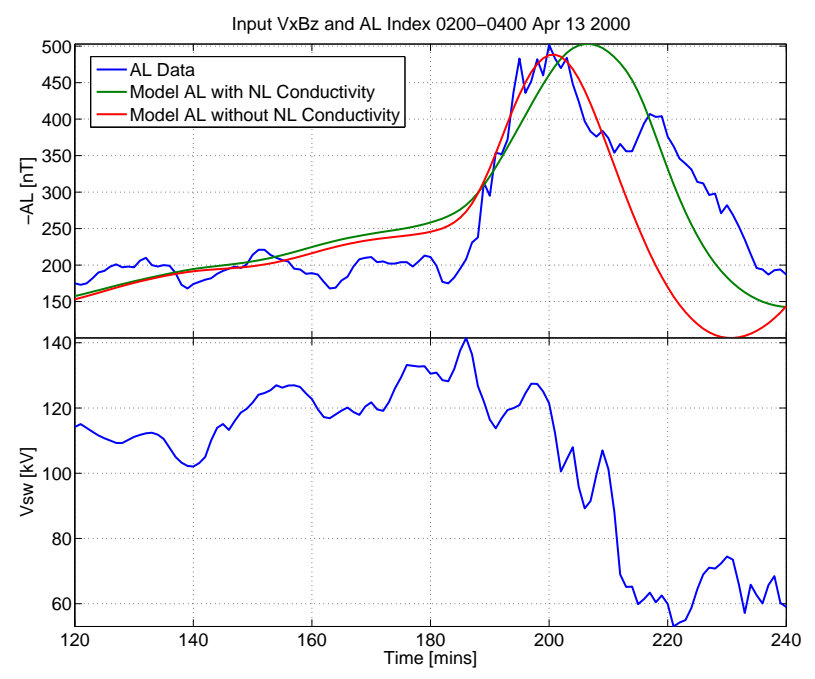

Fig. 3. 13 April 2000 substorm analysis with the WINDMI model. The result when using only a reconnection trigger $(\mathrm{ARV}=0.71)$ is compared to the result when conductivity enhancement $(\mathrm{ARV}=$ 0.32 ) is also included.

Another substorm that has similar features to the 13 April 2000 event is an earlier event that occurred on 31 July 1997 . The onset for this substorm was timed at 02:40 UTC on the day (Aksnes et al., 2002). In this case we used OMNI data for the solar wind input parameters, obtained from the WIND satellite. The input rectified voltage is shown in the bottom panel of Fig. 4. For this substorm, conductance maps were generated in Aksnes et al. (2002) that clearly show the enhancement of conductivity between 21:00 MLT and 00:03 MLT as the substorm develops. In particular the Hall conductance showed a strong increase.

As before, we adjust our model in order to to fulfill two criteria. The first is that the onset time must be almost at the onset time reported. The second is that we try to capture the overall substorm growth, expansion and recovery phases. As in the previous case, the input voltage decreases sharply at approximately 03:05 UTC, which means that the observed recovery phase is not affected by solar wind driving. The IMF $\mathrm{Bz}$ remains southward during the onset, ensuring again that this substorm is not a case that is possibly driven by northward turning of the IMF.

Because the conductivity was in fact enhanced according to the reported values in Aksnes et al. (2002), we increased the saturation level of the conductivity to $\Sigma_{\text {enh }}=50$ and obtained a result that gave an ARV of 0.55 compared to the case when only the reconnection trigger is present, which gave an ARV of 1.06. The improvement is not significant from a qualitative point of view, but we can see in Fig. 4 that the green curve, representing the model output with enhanced conductivity, somewhat follows the almost square-like features of 


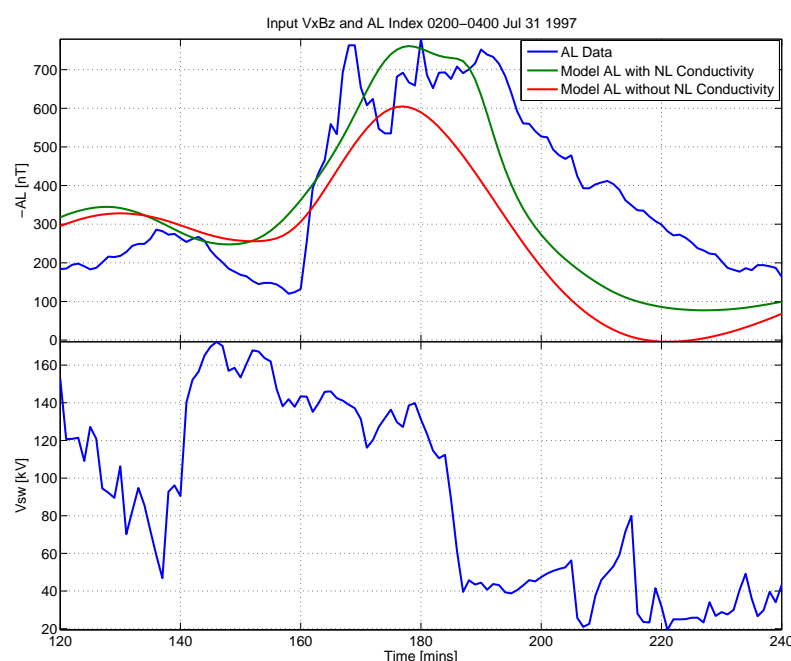

Fig. 4. 31 July 1997 substorm analysis with the WINDMI model. The result when using only a reconnection trigger $(\mathrm{ARV}=1.06)$ is compared to the result when conductivity enhancement $(\mathrm{ARV}=$ $0.55)$ is also included.

the AL peak. Increasing the conductivity level further did not improve the calculated ARV.

\section{Conclusions}

In this paper, we modified our nonlinear model of the magnetosphere to account for the enhanced ionospheric conductivity during substorm growth, onset and expansion. This is incorporated into the model by introducing a term in the equation driving the auroral electrojet that depends on the rate of particle flow along the magnetic field lines that begin in the plasma sheet and close in the higher latitude ionosphere.

We explored the most significant implications of the new term on the dynamics of the model. In addition, we used two substorm datasets, chosen because they were not triggered by northward turning of the IMF $\mathrm{Bz}$, and that the solar wind driver turns off during the recovery phase.

We found that the dynamics of the auroral electrojet is modified, depending on the level of enhancement, the energy content of the parallel flow of particles, and the ionization efficiency, which are controllable parameters in the model. The effect is most pronounced if the conductivity enhancement is sudden. For a gradual buildup and decay of conductivity, the electrojet current decays more slowly during the substorm recovery period, and does not overshoot negatively at the end of the substorm.

In future work, we will use the new model to analyze a larger dataset of geomagnetic substorms, such as that provided by the SuperMAG database (http:supermag.jhuapl. edu/).
Acknowledgements. The authors acknowledge WDC Kyoto for providing the AL index data and the NASA Space Physics Data facility (SPDF) for providing ACE spacecraft data.

Edited by: A. Surjalal Sharma

Reviewed by: M. Freeman, K. Unnikrishnan,

and one anonymous referee

\section{References}

Ahn, B.-H., Richmond, A., Kamide, Y., Kroehl, H., Emery, B., de la Beaujardie, O., and Akasofu, S.-I.: An ionospheric conductance model based on ground magnetic disturbance data, J. Geophys. Res., 103, 14769-14780, 1998.

Akasofu, S.: Several "Controversial” Issues on Substorms, Space Sci. Rev., 113, 1-40, 2004.

Aksnes, A., Stadsnes, J., Bjordal, J., Østgaard, N., Vondrak, R. R., Detrick, D. L., Rosenberg, T. J., Germany, G. A., and Chenette, D.: Instantaneous ionospheric global conductance maps during an isolated substorm, Ann. Geophys., 20, 1181-1191, doi:10.5194/angeo-20-1181-2002, 2002.

Baker, D., Pulkkinen, T., Buchner, J., and Klimas, A.: Substorms: A global instability of the magnetosphere-ionosphere system, J. Geophys. Res., 104, 14601-14611, 1999.

Birn, J., Artemyev, A., Baker, D., Echim, M., Hoshino, M., and Zelenyi, L.: Particle acceleration in the magnetotail and aurora, Space Sci. Rev., 173, 49-102, doi:10.1007/s11214-012-9874-4, 2012.

Blanchard, G. and McPherron, R.: A bimodal representation of the response function relating the solar wind electric field to the $\mathrm{AL}$ index, J. Adv. Space Res., 13, 4, 71-74, 1993.

Coumans, V., Gérard, J.-C., Hubert, B., Meurant, M., and Mende, S. B.: Global auroral conductance distribution due to electron and proton precipitation from IMAGE-FUV observations, Ann. Geophys., 22, 1595-1611, doi:10.5194/angeo-22-1595-2004, 2004.

Doxas, I., Horton, W., Lin, W., Seibert, S., and Mithaiwala, M.: A Dynamical Model for the Coupled Inner Magnetosphere and Tail, IEEE Trans. Plasma Sc., 32, 1443-1448, 2004.

Gallardo-Lacourt, B., Nishimura, Y., Lyons, L. R., and Donovan, E.: External triggering of substorms identified using modern optical versus geosynchronous particle data, Ann. Geophys., 30, 667673, doi:10.5194/angeo-30-667-2012, 2012.

Gjerloev, J. and Hoffman, R.: Height-integrated conductivity in auroral substorms: 1. Data, J. Geophys. Res., 105, doi:10.1029/1999JA900354, 2000a.

Gjerloev, J. and Hoffman, R.: Height-integrated conductivity in auroral substorms: 2. Modelling, J. Geophys. Res., 105, doi:10.1029/1999JA900353, 2000b.

Horton, W. and Doxas, I.: A low-dimensional energy-conserving state space model for substorm dynamics, J. Geophys. Res., 101, 27223-27237, 1996.

Horton, W., Weigel, R. S., Vassiliadis, D., and Doxas, I.: Substorm classification with the WINDMI model, Nonlin. Processes Geophys., 10, 363-371, doi:10.5194/npg-10-363-2003, 2003.

Huang, C., Foster, J., Goncharenko, L., Reeves, G., Chau, J., Yumoto, K., and Kitamura, K.: Variations of low-latitude geomagnetic fields and Dst index caused by magnetospheric substorms, J. Geophys. Res., 109, doi:10.1029/2003JA010334, 2004. 
Klimas, A., Baker, D., Vassiliadis, D., Roberts, D., Fairfield, D., and Buchner, J.: A nonlinear analog model of geomagnetic activity, J. Geophys. Res., 97, 1992.

Klimas, A., Vassiliadis, D., Baker, D., and Roberts, D.: The organized nonlinear dynamics of the magnetosphere, J. Geophys. Res., 101, A6, 13089-13113, 1996.

Klimas, A., Valdivia, J., Vassiliadis, D., and Baker, D.: AL index prediction using data-derived nonlinear prediction filters, Phys. Plasma., edited by: Chang, T. and Jasperse, J. R., 1998.

Knight, S.: Parallel electric fields, Planet. Space Sci., 21, 741-750, 1973.

Mays, M. L., Horton, W., Spencer, E., and Kozyra, J.: Real-time predictions of geomagnetic storms and substorms: Use of the Solar Wind Magnetosphere-Ionosphere System model, Space Weather, 7, doi:10.1029/2008SW000459, 2009.

Morioka, A., Miyoshi, Y., Miyashita, Y., an H. Misawa, Y. K., Tsuchiya, F., Kataoka, R., Kadokura, A., Mukai, T., Yumoto, K., Menietti, D., parks, G., Liou, K., Honary, F., and Donovan, E.: Two-step evolution of auroral acceleration at substorm onset, J. Geophys. Res., 115, doi:10.1029/2010JA015361, 2010.

Partamies, N., Pulkkinen, T., McPherron, R., McWilliams, K., Bryant, C., Tanskanen, E., Singer, H., Reeves, G., and Thomsen, M.: Statistical survey on sawtooth events, SMCs and isolated substorms, J. Adv. Space Res., 376-384, doi:10.1016/j.asr.2009.03.013, 2009.

Patra, S., Spencer, E., Horton, W., and Sojka, J.: Study of Dst/ring current recovery times using the WINDMI model, J. Geophys. Res., 116, doi:10.1029/2010JA015824, 2011.

Pulkkinen, A., Klimas, A., Vassiliadis, D., and Uritsky, V.: Role of stochastic fluctuations in the magnetosphere-ionosphere system: A stochastic model for the AE index variations, J. Geophys. Res., 111, doi:10.1029/2006JA011661, 2006.
Rasmussen, C., Schunk, R., and Wickwar, V.: A photochemical equilibrum model for ionospheric conductivity, J. Geophys. Res., 93, 9831-9840, 1988.

Reiff, P. H. and Luhmann, J. G.: Solar wind control of the polarcap voltage, in: Solar Wind-Magnetosphere Coupling, edited by: Kamide, Y. and Slavin, J. A.,453-476, Terra Sci., Tokyo, 1986.

Robinson, R., Vondrak, R., Miller, K., Dabbs, T., and Hardy, D.: On calculating ionospheric conductances from the flux and energy of precipitating electrons, J. Geophys. Res., 92, 2565-2669, 1987.

Sergeev, V., Angelopoulos, V., and Nakamura, R.: Recent advances in understanding substorm dynamics, Geophys. Res. Lett., 39, doi:10.1029/2012GL050859, 2012.

Spencer, E., Horton, W., Mays, L., Doxas, I., and Kozyra, J.: Analysis of the 3-7 October 2000 and 15-24 April 2002 geomagnetic storms with an optimized nonlinear dynamical model, J. Geophys. Res., 112, doi:10.1029/2006JA012019, 2007.

Tang, B., Wang, C., Hu, Y., and Kan, J.: Intensification of the Cowling current in the global MHD simulation model, J. Geophys. Res., 116, doi:10.1029/2010JA016320, 2011.

Vassiliadis, D., Klimas, A., Baker, D., and Roberts, D.: A description of solar-wind magnetosphere coupling based on nonlinear filters, J. Geophys. Res., 13, 100, A3, 3495-3512, 1995.

Weigel, R., Horton, W., Tajima, T., and Detman, T.: Forecasting auroral electrojet activity from solar wind input with neural networks, Geophys. Res. Lett., 26, 1353-1356, 1999.

Yoon, P., Lui, A., and Sitnov, M.: Generalized lower-hybrid drift instabilities in current sheet equilibrium, Phys. Plasma., 9, 15261538, 2002. 